\title{
Walking spinals: a myth or reality?
}

$\mathrm{T}$ is over a century since Augustus Bier introduced spinal anesthesia in 1899. It is still widely used in the developing countries because it is cheap, reliable and effective. I ts popularity in the western civilization was marred by the high incidence of post dural puncture headache (PDPH) until pencil point small gauge spinal needles were introduced. There is resurgence of enthusiasm with spinal anesthesia in many areas including cardiac surgery. I ts beneficial role in patients having lower limb orthopedic and vascular surgery is well established. Acceptance of this technique is far from a reality in the ambulatory setting. The fundamental problems are delayed return of bladder function and motor power as well as the dorsal column function in the lower limb, all of which can delay discharge in a patient who is not "sore, sick and sleepy". The introduction of newer general anesthetic agents that allow a predictable rapid emergence such as desflurane and sevoflurane also allow precise titration of anesthesia to the surgical length and requirement. This has deterred many a regional enthusiast from adopting spinal anesthesia for ambulatory surgery. A number of investigators have used modifications such as use of a smaller dose of local anesthetic, ${ }^{1,2}$ addition of opioids as adjuvants, ${ }^{3}$ shorter acting local anesthetic such as procaine, ${ }^{4}$ unilateral spinal anesthesia ${ }^{2,5}$ and pure opioid for the spinal mainly in the obstetrical population. Although a number of these studies have actually documented reduction in motor block and earlier recovery adequate for ambulating, none of them have actually objectively evaluated patients' ability to walk out of the operating room, "a step ahead" compared to general anesthesia. In a few of these studies that compared the specified technique with general anesthesia, there was significant delay both with initiating the anesthetic as well as in discharge from the post anesthesia care unit in the spinal group.

Slow bolus of small doses of "hypobaric" bupivacaine" have been used to produce segmental analgesia to relieve intractable angina with implantable pumps while retaining the capacity to ambulate. ${ }^{6} \mathrm{C}$ an this be extrapolated to surgical anesthesia? H ow low a dose can one give to accomplish adequate duration and intensity of anesthesia for a given surgery? Liu et al. ${ }^{7}$ looked at the dose response to $3.75 \mathrm{mg}, 7.5 \mathrm{mg}$ and $11.25 \mathrm{mg}$ of bupivacaine for spinal anesthesia and note that each additional mg of bupivacaine spinally translates to prolongation of sensory block to transcutaneous electrical stimulation by ten minutes, motor block by eight minutes and recovery time by $21 \mathrm{~min}$. L iew et al. ${ }^{8}$ have used $25 \mathrm{mg}$ of $0.5 \%$ lidocaine (hypobaric) for outpatient gynecological surgery with motor blocks that resolved within an hour. H ow much lower can one go with dosing without affecting efficacy? In this issue Vaghadia et al. have given us this crucial information using population pharmacodynamic modeling, clinical evaluation and pharmacoeconomic comparison using selective spinal anesthesia for outpatient laparoscopy. Albeit in a small number, they were able to use selective spinal anesthesia for very short duration abdominal surgery and yet manage accomplishing discharge times comparable to general anesthesia. They have also shown that pure opioid spinal anesthesia is insufficient for surgical anesthesia for gynecological laparoscopy whereas it has been shown to provide adequate analgesia in the obstetric population. Did they use this "ultra light" spinal anesthesia as a component of balanced anesthesia? In their paper comparing sufentanil (S) to lidocaine/ sufentanil mixture (LS), six of the seven patients in the LS group had reported mild to moderate abdominal or shoulder discomfort and one requiring conversion to general anesthesia but the patients were all satisfied except the ones that had to be converted to general anesthesia.

$C$ an this technique work in every anesthesiologists' hands? Will this be adequate for lower limb surgery? Did the technique accomplish a purely segmental abdominal light sensory and sympathetic block? From their data it is obvious that their patients did get sensory blockade of the sacral segments. O bviously the fast recovery could only be attributed to the lowest 
dose of intrathecal lidocaine and opioid, the latter intensifying the sensory block for its duration. $\mathrm{H}$ ow does this technique compare to local infiltration and sedation for laparoscopic surgery? U se in a larger population by the anesthesia community and future clinical trials certainly will give us more insight into the efficacy of the selective spinal anesthesia for ambulatory surgery. That is what will determine if "walking spinals are a myth or a reality" for ambulatory surgery. For now, this is the greatest stride we have made with the spinals.

\section{La rachianesthésie ambulatoire : un mythe ou une réalité?}

II y a un peu plus d'un siècle, soit en 1899, Augustus Bier faisait connaître la rachianesthésie. Cette technique est encore très utilisée dans les pays en développement, car elle est bon marché, fiable et efficace. Sa popularité dans les pays occidentaux a été assombrie par la forte incidence de céphalées postponction durale (CPPD) jusqu'à ce que des aiguilles de petit calibre à pointe effilée ne fassent leur apparition. Il y a donc un regain d'enthousiasme pour la rachianesthésie dans de multiples domaines, y compris la cardiochirurgie. Son rôle bénéfique chez les patients qui subissent une intervention orthopédique et vasculaire aux membres inférieurs est bien connu. $M$ ais cette technique est loin d'être acceptée dans le contexte ambulatoire. Les complications principales sont la récupération retardée du tonus vésical et de la fonction motrice de même que des foncions de la corne postérieure liées aux membres inférieurs, ce qui peut prolonger le séjour hospitalier d'un patient qui, par ailleurs est non souffrant, non malade ou non somnolent. L'arrivée de nouveaux agents pour l'anesthésie générale, comme le desflurane et le sévoflurane qui permettent un réveil rapide prévisible, permettent aussi un dosage précis de l'anesthésie selon les besoins ou la durée de l'intervention chirurgicale. La situation en a découragé bon nombre d'adopter la rachianesthésie en chirurgie ambulatoire. Certains chercheurs ont apporté des modifications comme l'usage de plus petites doses d'anesthésique local, ${ }^{1,2}$ l'addition d'opioïdes comme adjuvants, ${ }^{3}$ I'utilisation des anesthésiques locaux d'action plus brève comme la procaïne, ${ }^{4}$ la réalisation d'une rachianesthésie unilatérale 2,5 et l'emploi d'opioïde pur principalement pour la rachianesthésie obstétricale. Certaines des études ont fait la preuve d'une réduction du blocage moteur et de la récupération plus rapide des patients ambulatoires, mais aucune d'elles n'a vraiment évalué objectivement la capacité de quitter la salle d'opération, un "pas en avant" sur l'anesthésie générale. Des études ont comparé cette technique à l'anesthésie générale et ont montré un délai significatif du début d'action de l'anesthésique et un départ retardé de la salle de réveil chez les patients sous rachianesthésie.

Des petites doses de bupivacaïne "hypobare" ont été administrées en bolus lents avec des perfusions implantables pour produire une analgésie segmentaire visant à soulager une douleur angineuse rebelle, tout en conservant la capacité de marcher. ${ }^{6}$ Q uelle est la plus faible dose possible permettant de produire une anesthésie de durée et d'intensité adéquates pour une intervention chirurgicale donnée? Liu et coll. ${ }^{7}$ ont observé les réactions à des doses de $3,75 \mathrm{mg}, 7,5 \mathrm{mg}$ et $11,25 \mathrm{mg}$ de bupivacaïne pour la rachianesthésie et ont noté que pour chaque mg du médicament ajouté, il y a une prolongation du bloc sensitif rachidien de dix minutes, à l'électrostimulation transcutanée, du bloc moteur de huit minutes et de la récupération de $21 \mathrm{~min}$. L iew et coll. ${ }^{8}$ ont utilisé $25 \mathrm{mg}$ de lidocaïne (hypobare) à $0,5 \%$ pour une intervention gynécologique ambulatoire. Les blocs moteurs ont été renversés en moins d'une heure. Jusqu'où peut-on encore réduire les doses sans affecter l'efficacité? Dans le présent numéro, Vaghadia et coll. nous fournissent cette importante information en utilisant la modélisation pharmacodynamique d'une population, l'évaluation clinique et la comparaison pharmaco-économique dans le cas de laparoscopies ambulatoires sous rachianesthésie. $M$ algré un nombre restreint de sujets, ils ont pu utiliser la rachianesthésie pendant une intervention abdominale de très courte durée et, néanmoins, réussir à donner congé dans des temps comparables à ceux de l'anesthésie générale. Ils ont aussi montré que l'opioïde pur est insuffisant pour la rachianesthésie pendant la laparoscopie gynécologique tandis qu'il suffit à l'analgésie pendant l'accouchement. O nt-ils utilisé cette rachianesthésie "ultra-légère" comme composante d'une anesthésie balancée? L ors de leur expérimentation comparant l'effet du sufentanil (S) à celui d'un mélange de lidocaïne et de sufentanil (LS), six des sept patientes du groupe LS ont rapporté un malaise abdominal de léger à modéré ou une gêne à l'épaule et une patiente a dû recevoir une anesthésie générale. Toutes, sauf la dernière, ont donc été satisfaites de la rachianesthésie. 
Cette technique peut-elle réussir pour tous les anesthésiologistes? Est-elle adéquate pour une intervention aux membres inférieurs? La technique produit-elle seulement un blocage sensitif et sympathique segmentaire léger? Selon les données des auteurs, il est évident que leurs patientes $n$ 'ont pas subi de bloc sensitifs segmentaires sacrés. Sans aucun doute, la récupération rapide ne peut être attribuée qu'à la plus faible dose intrathécale de lidocaïne et d'opioïde, la dernière intensifiant le bloc sensitif pour la durée de son action. Comment se compare cette technique avec I'infiltration locale et la sédation pendant la laparoscopie chirurgicale? L'utilisation par une plus grande partie des anesthésiologistes et la réalisation de nouveaux essais nous donneront certainement une meilleure idée de l'efficacité de la rachianesthésie sélective en chirurgie ambulatoire. C'est ce qui déterminera si "la rachianesthésie ambulatoire est un mythe ou une réalité" pour ce type d'interventions chirurgicales. Pour l'instant, c'est le plus important progrès réalisé en rachianesthésie.

\section{References}

1 Chan VWS, Garcia J, A I-K aisy A, D rasner K. A comparative study of low-dose hyperbaric spinal lidocaine $0.5 \%$ versus $5 \%$ for continuous spinal anesthesia. Reg Anesth Pain M ed 1998; 23: 164-9.

2 Kuusniemi KS, Pihlajamaki KK, Pitkanen MT. A low dose of plain or hyperbaric bupivacaine for unilateral spinal anesthesia. Reg Anesth Pain M ed 2000; 25: 605-10.

3 K uusniemi KS, Pihlajamaki KK, Pitkanen MT, $\mathrm{H}$ eleni us $\mathrm{H} Y, \mathrm{~K}$ irvela $\mathrm{OA}$. The use of bupivacaine and fentanyl for spinal anesthesia for urologic surgery. Anesth Analg 2000; 91: 1452-6.

4 Mulroy M F, Larkin KL, H odgson PS, $\mathrm{H}$ elman JD, Pollock JE, Liu SS. A comparison of spinal, epidural, and general anesthesia for outpatient knee arthroscopy. Anesth Analg 2000; 91: 860-4.

5 Casati A, Fanelli G, Cappelleri G, Borghi B, Cedrati V, Torri G. Low dose hyperbaric bupivacaine for unilateral spinal anaesthesia. Can J Anaesth 1998; 45: 850-4.

6 Dahm P, N itescu P, A ppelgren L, Curelaru I. H igh thoracic/ low cervical, long-term intrathecal (i.t) infusion of bupivacaine alleviates "refractory" pain in patients with unstable angina pectoris. Report of 2 cases. Acta Anaesthesiol Scand 1998; 42: 1010-7.

7 Liu SS, Ware PD, Allen H W, N eal JM, Pollock JE. D ose-response characteristics of spinal bupivacaine in volunteers. Clinical implications for ambulatory anesthesia. Anesthesiology 1996; 85: 729-36.

8 Liew QY, Tay D H, ThomasE. Lignocaine $0.5 \%$ for spinal anaesthesia in gynaecological day surgery. Anaesthesia 1994; 49: 633-6.
9 Vaghadia H, Collins L, Sun H, Mitchel GWE. Selective spinal anesthesia for outpatient laparoscopy. IV: Population pharmacodynamic modeling. Can J Anesth 2001; 48: 273-78.

10 Vaghadia H, Viskari D, Mitchell GWE, Burrill A. Selective spinal anesthesia for outpatient laparoscopy. I: Characteristics of three hypobaric solutions. Can J Anesth 2001; 48: 256-60. 